\title{
Risk Factors Associated with Development of Acute and Sub-Acute Post-Cesarean Pain: A Prospective Cohort Study
}

This article was published in the following Dove Press journal: Journal of Pain Research

\author{
Jason Ju In Chan' \\ Chin Wen Tan $\mathbb{D}^{1,2}$ \\ Cheng Teng Yeam (iD) ${ }^{2}$ \\ Rehena Sultana ${ }^{3}$ \\ Alex Tiong Heng Sia ${ }^{1,2}$ \\ Ashraf Samir Habib (iD) ${ }^{4}$ \\ Ban Leong Sng ${ }^{1,2}$ \\ 'Department of Women's Anesthesia, \\ KK Women's and Children's Hospital, \\ Singapore; ${ }^{2}$ Anesthesiology and \\ Perioperative Sciences Academic Clinical \\ Program, Duke-NUS Medical School, \\ Singapore; ${ }^{3}$ Centre for Quantitative \\ Medicine, Duke-NUS Medical School, \\ Singapore; ${ }^{4}$ Department of \\ Anesthesiology, Duke University, \\ Durham, NC, USA
}

Background: Although cesarean delivery is one of the most commonly performed surgical procedures, robust data regarding post-cesarean pain is still lacking. Recent studies showed an association between pain upon local anesthetic (LA) injection for spinal anesthesia, or the use of a "three simple questions", and acute post-cesarean pain. Nevertheless, these assessments have yet to be validated further, despite their relative ease of use. We aimed to assess the association between pain score upon LA injection with acute post-cesarean pain after 24 hours at rest (primary outcome) and sub-acute post-cesarean pain lasting for 4 weeks or more (secondary outcome).

Methods: Women undergoing cesarean delivery under spinal anesthesia were given preoperative questionnaires on pain and psychological vulnerability. We also assessed the pain score upon LA injection and mechanical temporal summation. Univariate and multivariable logistic regressions were performed.

Results: The incidence of moderate-to-severe acute post-cesarean pain at 24 hours was $21.0 \%$ (95\% CI=16.6-27.6\%) (48 of 217 patients). Pain score upon LA injection was not significantly associated with acute post-cesarean pain after 24 hours at rest (unadjusted $\mathrm{OR}=1.10,95 \% \mathrm{CI}=0.95-1.27, P=0.21$ ). However, pain score upon LA injection was significantly associated with sub-acute post-cesarean pain (adjusted OR=1.29, 95\% $\mathrm{CI}=1.07-1.55, P=0.0089)$ with significant covariate of increased pre-operative central sensitization inventory $(\mathrm{CSI})$ scores (adjusted $\mathrm{OR}=1.05,95 \% \mathrm{CI}=1.01-1.09, P=0.0111$; area under the curve $(\mathrm{AUC})=0.691)$.

Conclusion: There was no association between increased pain score upon LA injection and acute post-cesarean pain, but it was associated with sub-acute post-cesarean pain. Further work is needed to define pain score upon LA injection as a convenient pragmatic measure of risk stratifying patients predisposed to sub-acute post-cesarean pain.

Keywords: obstetrics, pain measurement, pain outcome measurement, post-operative pain

\section{Background}

Cesarean delivery is one of the most common surgical procedures performed in the United States, making up over $32 \%$ of all live births - about 1.3 million per year. ${ }^{1}$ Among these patients, approximately $20 \%$ of women will experience severe, acute pain after cesarean delivery. ${ }^{2}$ Severe acute post-cesarean pain is found to increase the risk of developing postnatal depression and persistent pain, ${ }^{3,4}$ with the latter occurring in $9.2-18 \%$ of women after cesarean delivery. ${ }^{5-7}$ Given that inter-individual pain experience is highly variable, it is imperative to optimize and individualize pain management strategies during the post-operative period.
Department of Women's Anesthesia, KK

Women's and Children's Hospital, 100

Bukit Timah Road, 229899, Singapore

Tel +656394 I08I

Fax +65 6291266I

Email sng.ban.leong@singhealth.com.sg
Journal of Pain Research 2020:13 2317-2328 
Studies investigating post-cesarean pain have investigated a combination of demographic, anesthetic, maternal, obstetric factors, among others. ${ }^{8}$ For instance, higher pre-operative anxiety was found to correlate with post-cesarean delivery analgesic needs, ${ }^{9}$ while a high preoperative pain catastrophizing score was associated with higher post-cesarean pain scores. ${ }^{10}$ Local anesthetic (LA) injection is part of the standard practice during neuraxial anesthesia. Orbach-Zinger et $\mathrm{al}^{11}$ demonstrated that increased pain upon LA injection for spinal anesthesia is associated with increased acute post-cesarean pain scores. Similarly, a more recent study using "three simple questions" (anxiety about upcoming surgery, anticipated pain score, and anticipated pain medication needs) showed that patient's responses were correlated with post-operative pain on mobilization in both derivation and validation cohorts, accounting for up to $20 \%$ of the variance in postCesarean pain. ${ }^{12}$ Mechanical temporal summation (MTS) has also been previously investigated as a potential quantitative sensory testing (QST) modality to predict pain. ${ }^{13}$ Despite the relative ease of use, LA injection, the "three simple questions", and MTS have yet to be validated further.

Longer term sub-acute pain after cesarean delivery and psychological concerns such as postnatal depression are important outcomes after patient discharge. ${ }^{14,15}$ This could be associated with persistent post-cesarean pain which is defined as pain beyond 2 months after surgery. ${ }^{16}$ In view of this, we conducted a prospective cohort study to assess the association between pain score upon LA injection and acute 24 hours post-cesarean pain at rest (primary outcome) and sub-acute post-cesarean pain (pain that lasted 4 weeks or more) (secondary outcome).

\section{Patients and Methods}

\section{Patient Recruitment}

This prospective cohort study was conducted between May 2018 and April 2019 at KK Women's and Children's Hospital, Singapore on women undergoing elective cesarean delivery under neuraxial anesthesia. The study was approved by the SingHealth Centralized Institutional Review Board, Singapore (Ref: 2017/2381), and registered on Clinicaltrials.gov (NCT03645239). Written informed consent was obtained from women aged 21-50 years old, 36 weeks gestation or more, and with American Society of Anesthesiologist (ASA) physical status of I or II. Patients with a history of intravenous drug or opioid abuse, previous history of chronic pain syndrome, and patients undergoing emergency cesarean delivery or cesarean delivery under general anesthesia were excluded. Women who were unable to communicate in English were also excluded from this study.

Pre-operative assessment included demographic data, pain scores at rest or with movement $(0-10$; Numerical Rating Scale (NRS)) (Figure 1). The "three simple questions"12 were administered in the pre-operative assessment for i) Anxiety about upcoming surgery

On a scale of $0-100$, with 0 being not anxious at all and 100 being extremely anxious, how anxious are you about your upcoming surgery?

ii) Anticipated pain score

On a scale of $0-100$, with 0 being no pain at all and 100 the most severe pain that you could imagine, how much pain do you anticipate experiencing after your upcoming surgery?

And using a categorical scale for iii) Anticipated pain medication needs

On a scale of $0-5$, with 0 being none at all, and 5 being much more than average, how much pain medication do you anticipate needing after your upcoming surgery?

Mechanical temporal summation (MTS) was evoked using a 180-gram von Frey filament applied to the patient's forearm, as demonstrated by Weissman-Fogel et al, ${ }^{17}$ before anesthesia and surgery. After one touch, the subject was asked to rate the pinprick pain on a $0-100$ verbal numerical pain scale. Ten consecutive touches were then applied within a $1 \mathrm{~cm}$ diameter circle at 1 second interstimulus interval and the subject was asked to rate the pain of the 10th application. The difference between the last and the first pain scores were calculated, by which a positive difference $(>0)$ indicated the presence of an evoked MTS. Pre-operative questionnaires were administered after MTS assessment, which included: i) Central Sensitization Inventory (CSI), a questionnaire to quantify patients' reactions to expansion of the pain field and prolonged pain after stimulus removal; ${ }^{18}$ ii) Edinburgh Postnatal Depression Scale (EPDS), a self-reporting scale to assess postnatal depression; ${ }^{19}$ iii) Hospital Anxiety and Depression Scale (HADS) to assess the patients' level of anxiety and depression; ${ }^{20}$ and iv) Pain Catastrophizing Scale (PCS), a validated psychometric instrument to evaluate the negative thought processes patients may have 


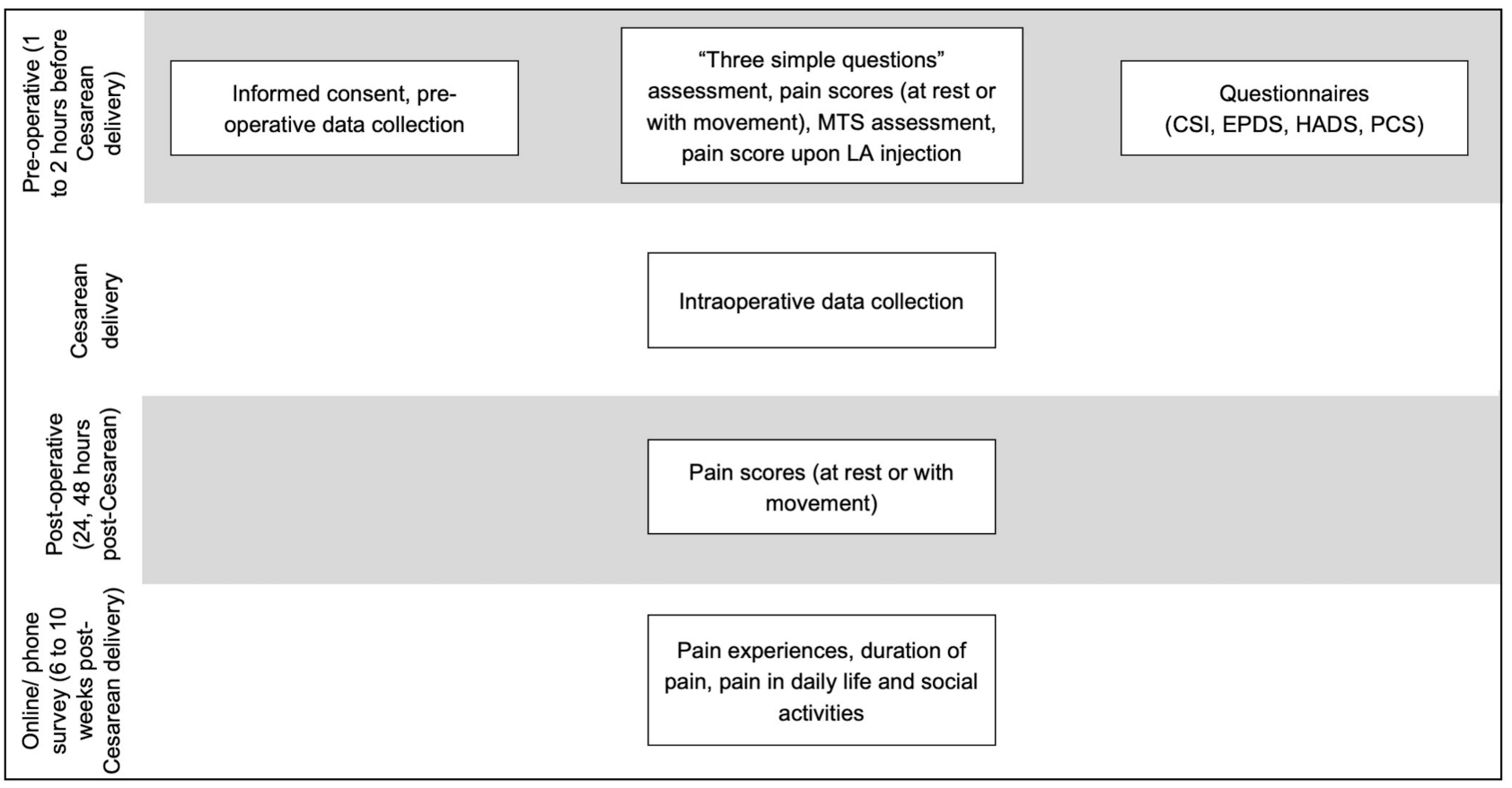

Figure I Study timeline.

Abbreviations: CSI, Central Sensitization Inventory; EPDS, Edinburgh Postnatal Depression Scale; HADS, Hospital Anxiety and Depression; LA, local anesthetic; MTS, mechanical temporal summation; PCS, Pain Catastrophizing Scale.

when they are exposed to actual or anticipated pain and painful experiences. ${ }^{21}$

Intraoperative procedures included routine standards of care of cesarean delivery in the hospital. All patients received antacid prophylaxis with $30 \mathrm{~mL}$ Sodium Citrate. Two to five milliliters of $1.0 \%$ lignocaine was used as the LA to the skin using a 22 Gauge hypodermic needle. A standardized script was used to inform patients when the LA was about to be given. After the LA injection, a NRS 0 to 10 was used to score the pain score upon LA injection. ${ }^{11}$ Both the LA injection and MTS assessments were performed by two formally trained investigators. Intra-operative and post-operative management was carried out at attending clinician's discretion based on hospital standards of care.

In brief, patients underwent either spinal or combined spinal-epidural (CSE) anesthesia during cesarean delivery, where the anesthesia procedures were all done by using $0.5 \%$ bupivacaine with dextrose additive. During the surgery, all patients were given spinal bupivacaine and fentanyl $(15 \mathrm{mcg})$ and morphine $(100 \mathrm{mcg})$ intrathecally as adjuncts for anesthesia. The testing and monitoring of spinal bloc were performed at different time points, including the presence of a motor block before incision, the absence of pain on surgical incision, and the absence of pain at the end of surgery. Blood pressure, three-lead ECG, pulse oximeter heart rate, and saturation were monitored throughout the duration of surgery. Blood loss, the presence of adhesion and intraoperative complications were also documented accordingly. In this study, all patients underwent a transverse incision.

During the postoperative period, resolution of spinal block height to $\mathrm{T} 4$ or below was required prior to discharge from the post-anesthesia care unit (PACU). Regular monitoring would be conducted in a general ward of hourly parameters including pain score, blood pressure, heart rate, and oxygen saturation. Postoperative analgesia (paracetamol, mefenamic acid, tramadol) were administered to patients after cesarean delivery, and tramadol would be administered as per patient request for excess pain. Pain scores at rest and with movement were documented at 24 and 48 hours after cesarean delivery.

An online survey was conducted at 6-10 weeks postcesarean delivery on the patient's pain experience and duration of pain. The presence of sub-acute post-cesarean pain (as defined by binary outcome presence of absence of pain that lasted for 4 weeks or more) at 6-10 weeks postcesarean delivery was recorded. Other pain-related questions were asked to look at the presence or absence of pain around the scar or abdominal wound site at greater than 4 
weeks, the time of resolution of pain, how pain affected the ability to walk, and how pain interfered with mood, sleep, concentration, and relationships.

\section{Sample Size Calculation and Statistical Analysis}

The calculated sample size of 196 patients was based on an incidence rate assumption of severe acute post-cesarean pain of $20 \% ;^{3}$ patients with pain score upon LA injection having severe acute post-cesarean pain with an anticipated odds ratio (OR) of 3.0; 5\% level of significance; $80 \%$ power, and 1:3 allocation ratio. After adjusting for $11 \%$ loss to follow-up, ineligibility, and withdrawal, the required sample size was at least 200 .

The acute post-cesarean pain after 24 hours at rest was collected as continuous data and ranged from 0 to 10 in NRS. The primary outcome of acute post-cesarean pain after 24 hours at rest was categorized into two categories "pain scores 0-3" or "pain scores 4-10", and was treated as a binary variable. We chose to use $0-3$ as one group as these patients would not need or be offered additional rescue medications, compared to moderate to severe pain having a pain score of $4-10$ in clinical practice. The secondary outcome sub-acute post-cesarean pain was also treated as a binary variable with categories "yes" or "no". All demographic, clinical, and anesthetic variables were summarized based on the primary outcome of acute postcesarean pain after 24 hours at rest and secondary outcome of sub-acute post-cesarean pain. Categorical variables were summarized as frequency (proportion), while continuous variables were summarized as mean \pm standard deviation (SD) or median (interquartile range (IQR)). Univariate and multivariable logistic regression analyses were performed to assess the association of the primary outcome with potential risk factors. Association from a logistic regression model was expressed as odds ratio (OR) and corresponding 95\% confidence interval (95\% CI). Multivariable logistic regression was then performed using those variables with a $P$-value $<0.02$ in the univariate logistic regression analysis. Then union of the variables from forward, backward, and stepwise method were used to finalize the variable lists in the multivariable model with entry and stay criteria as 0.02 and 0.05 , respectively. Then we used a likelihood ratio test followed by area under the curve (AUC) to decide the final multivariable model. Separate univariate and multivariable logistic regression analyses were performed to find associated independent factors for sub-acute post-cesarean pain. The significance level was set at 0.05 and all tests were two-tailed. SAS version 9.4 software (SAS Institute; Cary, NC, USA) was used for the analysis.

\section{Results}

A total of 218 women of ASA I-II were recruited in the study, however one patient withdrew prior to 24 hours follow-up (Figure 2). For the primary time point of acute post-cesarean pain at 24 hours at rest, 217 patients completed the follow-up. A further 12 patients were excluded from the analysis due to withdrawal, lost to follow-up, or not completing surveys at $6-10$ weeks post-cesarean delivery. The mean (SD) of the follow-up duration was 6.6 (0.9) weeks. At 24 hours post-cesarean delivery, 217 patients were categorized into two groups, with the first having minimal to mild pain scores of $0-3(n=169)$, and the other having moderate-to-severe pain scores of 4-10 $(\mathrm{n}=48)$. In this study, the incidence of moderate-to-severe acute post-cesarean pain at 24 hours at rest was $21.0 \%$ (95\% CI=16.6-27.6\%). Table 1 shows the pre-operative demographic data for the patients, and none of them was found to be associated with the acute post-cesarean pain except race, whereby Malay patients had an increased risk of having high acute 24 hours post-cesarean pain at rest as compared with Chinese $(\mathrm{OR}=2.22,95 \% \mathrm{CI}=1.03-4.77$, $P=0.0419$ ).

The majority of patients were given spinal anesthesia (195 or $89.9 \%$ ) with the remaining $22(10.1 \%)$ patients receiving a CSE anesthesia (Table 2). All patients underwent transverse surgical incision, with one from the moderate-to-severe acute post-cesarean pain group having failed neuraxial anesthesia, in which an epidural was inserted and top-up ropivacaine was given to achieve a block to T6 dermatome prior to incision. We found no significant association between blood loss and acute postcesarean pain score, or between surgery duration and acute post-cesarean pain score. A total of 35 patients were found to have adhesions with adhesiolysis done. A total of five patients were found to have intraoperative complications and showed no significant association with the severity of the acute post-cesarean pain score after 24 hours at rest.

Association of various measures with post-operative pain scores for up to 24 hours are displayed in Table 2. The primary outcome of the association between preoperative pain score upon LA injection and acute postcesarean pain 24 hours post-cesarean delivery was not significant $(\mathrm{OR}=1.10,95 \% \mathrm{CI}=0.95-1.27, P=0.2080)$. Of 


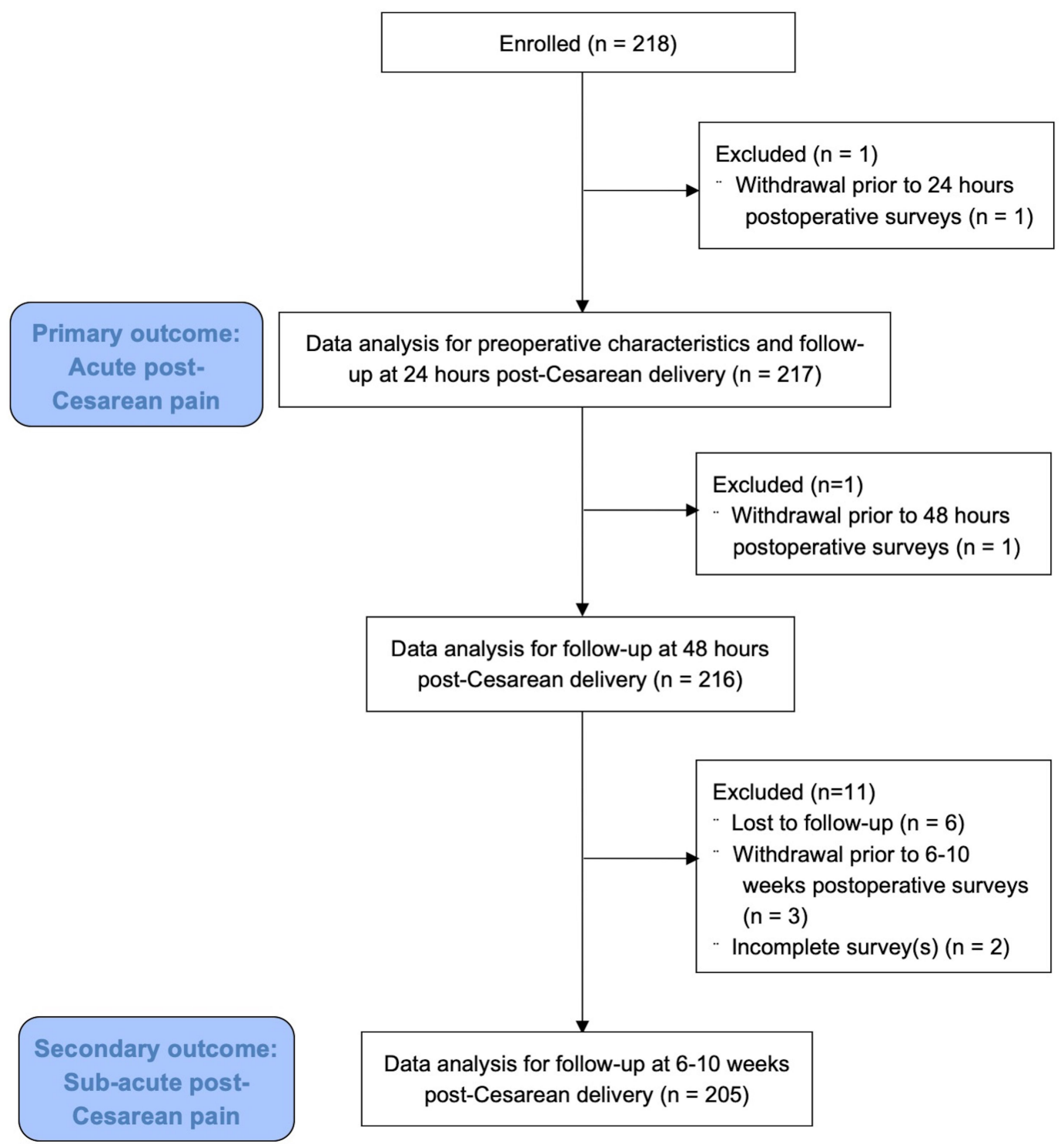

Figure 2 Study flowchart.

the "three simple questions" conducted pre-operatively, only anxiety about upcoming surgery was significantly associated with moderate-to-severe acute post-cesarean pain at 24 hours $(\mathrm{OR}=1.02,95 \% \quad \mathrm{CI}=1.01-1.04$, $P=0.0072$ ). The other two questions on anticipated pain scores $(\mathrm{OR}=1.02,95 \% \mathrm{CI}=1.00-1.04, P=0.0545)$ and anticipated pain medication requirements $(\mathrm{OR}=0.97$, 95\% CI $=0.66-1.43, P=0.8762$ ) were not significantly associated with acute post-cesarean pain at 24 hours. An evoked MTS (pinprick differences $>0) \quad(\mathrm{OR}=0.89,95 \%$ $\mathrm{CI}=0.45-1.76, P=0.7274)$, pain scores at rest $(\mathrm{OR}=1.07$, $95 \% \mathrm{CI}=0.78-1.50, P=0.6761)$, and pain scores with movement $\quad(\mathrm{OR}=1.05,95 \% \quad \mathrm{CI}=0.78-1.43, \quad P=0.7590)$ were also not significantly associated with moderate-to- severe acute post-cesarean pain at 24 hours. At 48 hours post-cesarean delivery, those with moderate-to-severe acute post-cesarean pain at 24 hours also exhibited greater pain score (at rest: $3.1 \pm 2.5$; with movement: $6.0 \pm 1.8$ ) than patients with minimal-to-mild acute post-cesarean pain (at rest: $1.4 \pm 1.8$; with movement: $4.5 \pm 2.1$ ). The use of intraoperative (morphine, fentanyl, paracetamol) and postoperative (paracetamol, mefenamic acid, tramadol) analgesia were not significantly associated with moderate-to-severe acute post-cesarean pain at 24 hours. Postoperative transversus abdominis plane (TAP) block was not performed.

Psychological assessments revealed that higher preoperative pain rumination scores $(\mathrm{OR}=1.09, \quad 95 \%$ $\mathrm{CI}=1.01-1.17, P=0.0359)$, magnification scores $(\mathrm{OR}=1.15$, 
Table I Demographic Characteristics (Based on 24 Hours Acute Post-Cesarean Pain Score, at Rest)

\begin{tabular}{|c|c|c|c|c|}
\hline Characteristics & Pain Scores 0-3 $(N=169)$ & Pain Scores 4-10 ( $N=48)$ & Unadjusted OR $(95 \% \mathrm{Cl})$ & $P$-value \\
\hline Age (years) & $34.8 \pm 5.0$ & $33.8 \pm 5.2$ & $0.96(0.89-1.03)$ & 0.2510 \\
\hline Race & & & & $0.2290^{\mathrm{a}}$ \\
\hline Chinese & $105(62.1)$ & $22(45.8)$ & 1 & - \\
\hline Malay & $31(18.3)$ & $15(31.3)$ & $2.22(1.03-4.77)$ & 0.0419 \\
\hline Indian & $18(10.7)$ & $6(12.5)$ & $1.58(0.56-4.42)$ & 0.3878 \\
\hline Others & $15(8.9)$ & $5(10.4)$ & $1.58(0.52-4.79)$ & 0.4227 \\
\hline Weight (kg) & $73.0 \pm 12.4$ & $74.1 \pm 12.2$ & $1.01(0.98-1.03)$ & 0.5857 \\
\hline Height (m) & $1.6 \pm 0.1$ & $1.6 \pm 0.1$ & $0.10(0.00-29.84)$ & 0.4307 \\
\hline BMI $\left(\mathrm{kg} / \mathrm{m}^{2}\right)$ & $28.8 \pm 4.6$ & $29.4 \pm 4.1$ & $1.03(0.96-1.11)$ & 0.3667 \\
\hline \multicolumn{5}{|l|}{ ASA physical status } \\
\hline I & $105(62.1)$ & $26(54.2)$ & I & - \\
\hline II & $64(37.9)$ & $22(45.8)$ & $1.35(0.7 \mathrm{I}-2.59)$ & 0.3586 \\
\hline Gestational age (weeks) & $38.3 \pm 0.8$ & $38.3 \pm 0.8$ & $0.90(0.45-1.77)$ & 0.7514 \\
\hline \multicolumn{5}{|l|}{ Past cesarean delivery } \\
\hline No & $56(33.1)$ & $17(35.4)$ & I & - \\
\hline Yes & $113(66.9)$ & $31(64.6)$ & $0.90(0.46-1.77)$ & 0.7680 \\
\hline
\end{tabular}

Notes: Values are expressed as mean \pm standard deviation (SD) or number (\%). ${ }^{\mathrm{a}}$ Type $3 P$-value.

Abbreviations: ASA, American Society of Anesthesiologists; BMI, body mass index; $\mathrm{Cl}$, confidence interval; OR, odds ratio.

95\% CI $=1.02-1.30, P=0.0255)$, and total pain catastrophizing scores $(\mathrm{OR}=1.04,95 \% \mathrm{CI}=1.00-1.07, P=0.0244)$ were associated with moderate-to-severe acute post-cesarean pain at 24 hours (Table 3). Pre-operative HADS depression $(\mathrm{OR}=0.99$, 95\% $\mathrm{CI}=0.89-1.10, P=0.8067)$, HADS anxiety $(\mathrm{OR}=1.08$, 95\% CI $=0.98-1.18, P=0.1293$ ), and EPDS (OR=1.03, 95\% $\mathrm{CI}=0.96-1.11, P=0.4447)$ did not show any significant association with acute post-cesarean pain at 24 hours.

From the follow-up pain survey at 6-10 weeks postcesarean delivery, the incidence of sub-acute post-cesarean pain, as defined by the presence of pain around their scar or abdominal wound site for 4 weeks or more, was $14.6 \%$ (30 of $205 ; 95 \% \mathrm{CI}=9.8-19.5 \%$ ). Out of these 30 patients, half of them $(n=15)$ had resolution of pain 4 weeks after delivery, while the remaining 15 had pain persisting beyond 4 weeks after delivery. In terms of daily life activities, $46.7 \%(\mathrm{n}=14)$ of these 30 patients reporting subacute post-cesarean pain also reported pain interfering with their ability to walk, followed by interference with $\operatorname{mood}(43.3 \%$ or $n=13)$, interference with sleep $(26.7 \%$ or $\mathrm{n}=8)$, interference with their relationships $(10 \%$ or $\mathrm{n}=3)$, and interference with their ability to concentrate $(6.7 \%$ or $\mathrm{n}=2)$. At $6-10$ weeks post-cesarean delivery, no significant differences in reasons for stopping breastfeeding were found between those with sub-acute post-cesarean pain, and patients without sub-acute post-cesarean pain. Eight out of $30(26.7 \%)$ patients with sub-acute post-cesarean pain had a greater EPDS score $\geq 10$; whereas in patients without sub-acute post-cesarean pain, 35 out of 175 patients $(20.0 \%)$ had a greater EPDS score $\geq 10$. There was no significant difference between patients with and without sub-acute post-cesarean pain $(P=0.4664)$ on the incidence of postpartum depression (defined as EPDS score $\geq 10$ ).

We did not find any multivariable model associated with pre-operative factors for acute post-cesarean pain at 24 hours post-cesarean delivery. All pre-operative variables and associated factors at 24 and 48 hours post-cesarean delivery were then analyzed for their associations with the presence of subacute post-cesarean pain at 6-10 weeks post-cesarean delivery, by which univariate factors with a $P$-value $<0.2$ are included in Table 4. Univariate analyses revealed that preoperative pain at rest $(\mathrm{OR}=1.71,95 \% \mathrm{CI}=1.14-2.57$, $P=0.0091)$ and with movement $\quad(\mathrm{OR}=1.41, \quad 95 \%$ $\mathrm{CI}=1.05-1.90, P=0.0228$ ), pain score upon LA injection $(\mathrm{OR}=1.27, \quad 95 \% \quad \mathrm{CI}=1.06-1.53, \quad P=0.0100)$, and preoperative central sensitization $\quad(\mathrm{OR}=1.05, \quad 95 \%$ $\mathrm{CI}=1.01-1.08, \quad P=0.0136)$ were significantly associated with the presence of sub-acute post-cesarean pain. Multivariable analysis showed that pre-operative pain score upon LA injection (adjusted OR=1.29, 95\% CI=1.07-1.55, $P=0.0089$ ) and pre-operative central sensitization (adjusted 
Table 2 Pain Characteristics and Univariate Analyses (Based on 24 Hours Acute Post-Cesarean Pain Score, at Rest)

\begin{tabular}{|c|c|c|c|c|}
\hline Characteristics & $\begin{array}{l}\text { Pain Scores } 0-3 \\
(N=169)\end{array}$ & $\begin{array}{l}\text { Pain Scores } 4-10 \\
(N=48)\end{array}$ & $\begin{array}{l}\text { Unadjusted OR }(95 \% \\
\mathrm{CI})\end{array}$ & $P$-value \\
\hline \multicolumn{5}{|l|}{ Pre-operative } \\
\hline Pain score (at rest; 0-10) & $0.1 \pm 0.9$ & $0.2 \pm 0.8$ & $1.07(0.78-1.50)$ & 0.6761 \\
\hline Pain score (with movement; 0-10) & $0.2 \pm 1.0$ & $0.3 \pm 1.0$ & $1.05(0.78-1.43)$ & 0.7590 \\
\hline Anxiety about upcoming surgery $(0-100)$ & $50[40.0]$ & $60[30.0]$ & $1.02(1.01-1.04)$ & 0.0072 \\
\hline Anticipated pain score $(0-100)$ & $70[20.0]$ & $77.5[10.0]$ & $1.02(1.00-1.04)$ & 0.0545 \\
\hline Anticipated pain medication needs $(0-5)$ & $3[0.0]$ & $3[1.0]$ & $0.97(0.66-1.43)$ & 0.8762 \\
\hline $\begin{array}{l}\text { Difference between eleventh and first pinprick } \\
\text { pain scores }\end{array}$ & $8.6 \pm 15.0$ & $9.1 \pm 12.4$ & $1.00(0.98-1.02)$ & 0.8167 \\
\hline \multicolumn{5}{|l|}{ Presence of evoked MTS } \\
\hline No & $52(30.8)$ & $15(31.9)$ & 1 & - \\
\hline Yes & 117 (69.2) & $32(68.1)$ & $0.89(0.45-1.76)$ & 0.7274 \\
\hline Pain score upon LA injection $(0-10)$ & $4.5 \pm 2.2$ & $5.0 \pm 2.3$ & $1.10(0.95-1.27)$ & 0.2080 \\
\hline $\begin{array}{l}\text { Number of patients with pain score upon LA } \\
\text { injection }>3\end{array}$ & $108(63.9)$ & $38(79.2)$ & $2.15(1.00-4.61)$ & 0.0501 \\
\hline \multicolumn{5}{|l|}{ Intra-operative and delivery } \\
\hline Neuraxial techniques & & & & 0.2524 \\
\hline CSE & $15(8.9)$ & $7(14.6)$ & 1.00 & - \\
\hline Spinal & $154(91.1)$ & $4 \mid(85.4)$ & $1.75(0.67-4.58)$ & - \\
\hline Surgery duration (mins) & $51.5 \pm 18.4$ & $53.7 \pm 18.9$ & I.0I (0.99-I.02) & $0.474 I$ \\
\hline Blood loss (mL) & $356.2 \pm 185.4$ & $389 \pm 334.3$ & $1.00(1.00-1.00)$ & 0.3934 \\
\hline Adhesions & $26(15.4)$ & $9(18.8)$ & $1.27(0.55-2.93)$ & 0.5764 \\
\hline Intraoperative complications & $4(2.4)$ & $\mathrm{I}(2.1)$ & $0.88(1.00-8.04)$ & $0.908 I$ \\
\hline Neonate ICU admission & $3(1.8)$ & $2(4.2)$ & $2.4 \mid(0.39-14.83)$ & $0.344 I$ \\
\hline \multicolumn{5}{|l|}{ Post-operative (24 hours) } \\
\hline Pain score (at rest; 0-10) & $0.8 \pm 1.1$ & $5.2 \pm 1.2$ & - & - \\
\hline Pain score (with movement; 0-10) & $3.9 \pm 2.2$ & $7.0 \pm 1.5$ & $2.20(1.73-2.80)$ & $<0.0001$ \\
\hline
\end{tabular}

Notes: Values are expressed as mean \pm standard deviation (SD), median [interquartile range (IQR)] or number (\%).

Abbreviations: Cl, confidence interval; LA, CSE, combined spinal-epidural; local anesthetic; MTS, mechanical temporal summation; OR, odds ratio.

$\mathrm{OR}=1.05,95 \% \mathrm{CI}=1.01-1.09, P=0.0111)$ were significantly associated with the presence of sub-acute post-cesarean pain at 6-10 weeks post-cesarean delivery. The area under curve (AUC) of ROC of this multivariable model was 0.691 (Figure 3).

\section{Discussion}

In this Asian population cohort study, pain score upon LA injection during spinal anesthesia administration was not associated with moderate-to-severe acute post-cesarean pain at rest 24 hours after cesarean delivery. However, this was associated with sub-acute post-cesarean pain at 6-10 weeks post-cesarean delivery survey. Increased pain score upon LA injection and increased pre-operative central sensitization were significant independent association factors of sub-acute post-cesarean pain.

Orbach-Zinger et $\mathrm{al}^{11}$ found that the use of pain score upon LA injection may be useful in predicting the severity of acute post-cesarean pain at 24 hours. Our finding showed no significant association between pain score upon LA injection and acute post-cesarean pain scores; however it was associated with sub-acute post-cesarean pain. The different study population and multiple operators using a pragmatic trial design could have accounted for differences in the findings. In addition, the evaluation of pain was performed using a NRS of 0-10 instead of 0-100, which may further lead to different interpretations as compared with Orbach-Zinger et al. ${ }^{11}$ Nevertheless, we found that pain score upon LA injection may affect subacute post-cesarean pain, which has important clinical implications in longer term post-cesarean pain management. Previous studies have reported the roles of ion channels in pain sensation. ${ }^{22}$ It is plausible that the increased pain during LA injection is attributed to ion channels of different acting responses, and hence the difference in mediating sub-acute pain instead of acute pain, 
Table 3 Psychological Characteristics and Univariate Analyses (Based on 24 Hours Acute Post-Cesarean Pain Score, at Rest)

\begin{tabular}{|l|l|l|l|l|}
\hline Characteristics & Pain Scores 0-3 (N=169) & Pain Scores 4-10 (N=48) & Unadjusted OR (95\% CI) & P-value \\
\hline \multicolumn{2}{|l|}{ Pre-operative } & $6.7 \pm 4.1$ & $1.09(1.01-1.17)$ & $1.15(1.02-1.30)$ \\
\hline PCS - Rumination (0-16) & $5.3 \pm 4.1$ & $4.0 \pm 3.1$ & $1.07(1.00-1.14)$ & 0.0359 \\
PCS - Magnification (0-12) & $3.1 \pm 2.4$ & $6.9 \pm 5.2$ & $1.04(1.01-1.07)$ & 0.0255 \\
PCS - Helplessness (0-24) & $5.4 \pm 4.4$ & $17.9 \pm 11.3$ & $1.00(0.97-1.02)$ & 0.0550 \\
PCS - Total Score (0-52) & $14.1 \pm 10.2$ & $52.8 \pm 11.2$ & $0.99(0.89-1.10)$ & 0.0244 \\
CSI (0-100) & $53.6 \pm 11.8$ & $4.1 \pm 2.9$ & $1.08(0.98-1.18)$ & 0.8067 \\
HADS - Depression (0-2I) & $4.3 \pm 3.1$ & $7.4 \pm 3.9$ & $1.03(0.96-1.11)$ & 0.1293 \\
HADS - Anxiety (0-2I) & $6.6 \pm 3.2$ & $7.5 \pm 4.3$ & 0.4447 \\
EPDS (0-30) & $7.0 \pm 4.2$ & &
\end{tabular}

Note: Values are expressed as mean \pm standard deviation (SD).

Abbreviations: $\mathrm{Cl}$, confidence interval; CSI, Central Sensitization Inventory; EPDS, Edinburgh Postnatal Depression Scale; HADS, Hospital Anxiety and Depression; OR, odds ratio; PCS, Pain Catastrophizing Scale.

Table 4 Factors Associated with Sub-Acute Post-Cesarean Pain at 6-10 Weeks Post-Cesarean Delivery

\begin{tabular}{|c|c|c|c|c|c|c|}
\hline \multirow[t]{2}{*}{ Parameters } & \multicolumn{2}{|c|}{$\begin{array}{l}\text { Presence of Sub-Acute Post- } \\
\text { Cesarean Pain }\end{array}$} & \multirow{2}{*}{$\begin{array}{l}\text { Unadjusted } \\
\text { OR }^{a} \\
(95 \% \mathrm{CI})\end{array}$} & \multirow[t]{2}{*}{$P$-value } & \multirow{2}{*}{$\begin{array}{l}\text { Adjusted } \\
\text { OR }^{\mathrm{b}} \\
(95 \% \mathrm{CI})\end{array}$} & \multirow[t]{2}{*}{ p-value } \\
\hline & No $(N=\mid 75)$ & Yes $(N=30)$ & & & & \\
\hline Intercept ( $\beta$ estimate (SE)) & - & - & - & - & $-5.57(1.21)$ & - \\
\hline Weight & $73.6 \pm 12.0$ & $69.3 \pm 15.0$ & $0.98(0.94-1.01)$ & 0.0844 & - & - \\
\hline Height & $1.6 \pm 0.1$ & $1.6 \pm 0.0$ & $0.01(0.00-5.69)$ & 0.1404 & - & - \\
\hline \multicolumn{7}{|l|}{ ASA physical status } \\
\hline 1 & III (63.4) & $15(50.0)$ & 1 & - & - & - \\
\hline II & $64(36.6)$ & $15(50.0)$ & $1.73(0.80-3.78)$ & 0.1659 & - & - \\
\hline Pre-operative pain score (at rest) & $0.1 \pm 0.5$ & $0.7 \pm 2.0$ & I.7I (1.14-2.57) & 0.0091 & - & - \\
\hline Pre-operative pain score (with movement) & $0.1 \pm 0.8$ & $0.7 \pm 1.8$ & $1.41(1.05-1.90)$ & 0.0228 & - & - \\
\hline Anticipated pain medication needs & $3.0[0.0]$ & $3.0[0.0]$ & $0.72(0.47-1.1 I)$ & 0.1393 & - & - \\
\hline Pain score upon LA injection & $4.5 \pm 2.1$ & $5.6 \pm 2.5$ & $1.27(1.06-1.53)$ & 0.0100 & $1.29(1.07-1.55)$ & 0.0089 \\
\hline CSI & $52.7 \pm 11.3$ & $58.4 \pm 12.3$ & $1.05(1.01-1.08)$ & 0.0136 & $1.05(1.01-1.09)$ & 0.0111 \\
\hline
\end{tabular}

Notes: Values are expressed as mean \pm standard deviation (SD), median [interquartile range (IQR)] or number (\%). ${ }^{\mathrm{a}} \mathrm{All}$ pre-operative variables and factors at $24-$ and 48 hours post-cesarean delivery were analyzed for their association with the presence of sub-acute post-cesarean pain at 6-10 weeks post-cesarean delivery, and only univariate factors with a $P$-value $<0.2$ are shown here. ${ }^{b}$ Adjusted $O R s$ were obtained from multivariate logistic regression by taking potential confounders $(P<0.02)$ identified by univariate analysis.

Abbreviations: ASA, American Society of Anesthesiologists; CI, confidence interval; CSI, Central Sensitization Inventory; LA, local anesthetic; OR, odds ratio; SE, standard error.

however further affirmation is needed. It is also notable that we found no significant association between acute post-cesarean pain and the incidence of sub-acute postcesarean pain at 6-10 weeks post-cesarean delivery, as opposed to a previous report on the positive correlation between acute pain and persistent pain after delivery. ${ }^{3}$ The knowledge of this information suggest variability in responses to pain among individuals, and that the use of pain score upon LA injection as a predictor on sub-acute post-cesarean pain may in turn aid in more personalized care and closer monitoring of the at-risk population to improve the pain management after discharge.
Central sensitization is characterized by allodynia, hyperalgesia, expansion of the pain field and prolonged pain after stimulus removal. ${ }^{18}$ Previous reports have demonstrated that depression and anxiety are often associated with central sensitization. ${ }^{23,24}$ However, further investigation on its relationship with persistent pain in cesarean delivery is still lacking. To our knowledge, this is the first study that utilized CSI in assessing central sensitization in patients undergoing cesarean delivery. We tried an interaction effect between CSI and preoperative HADS depression; and CSI and preoperative HADS anxiety separately. In both cases, interaction terms were highly insignificant $(P$-value $\geq 0.4)$ and there 


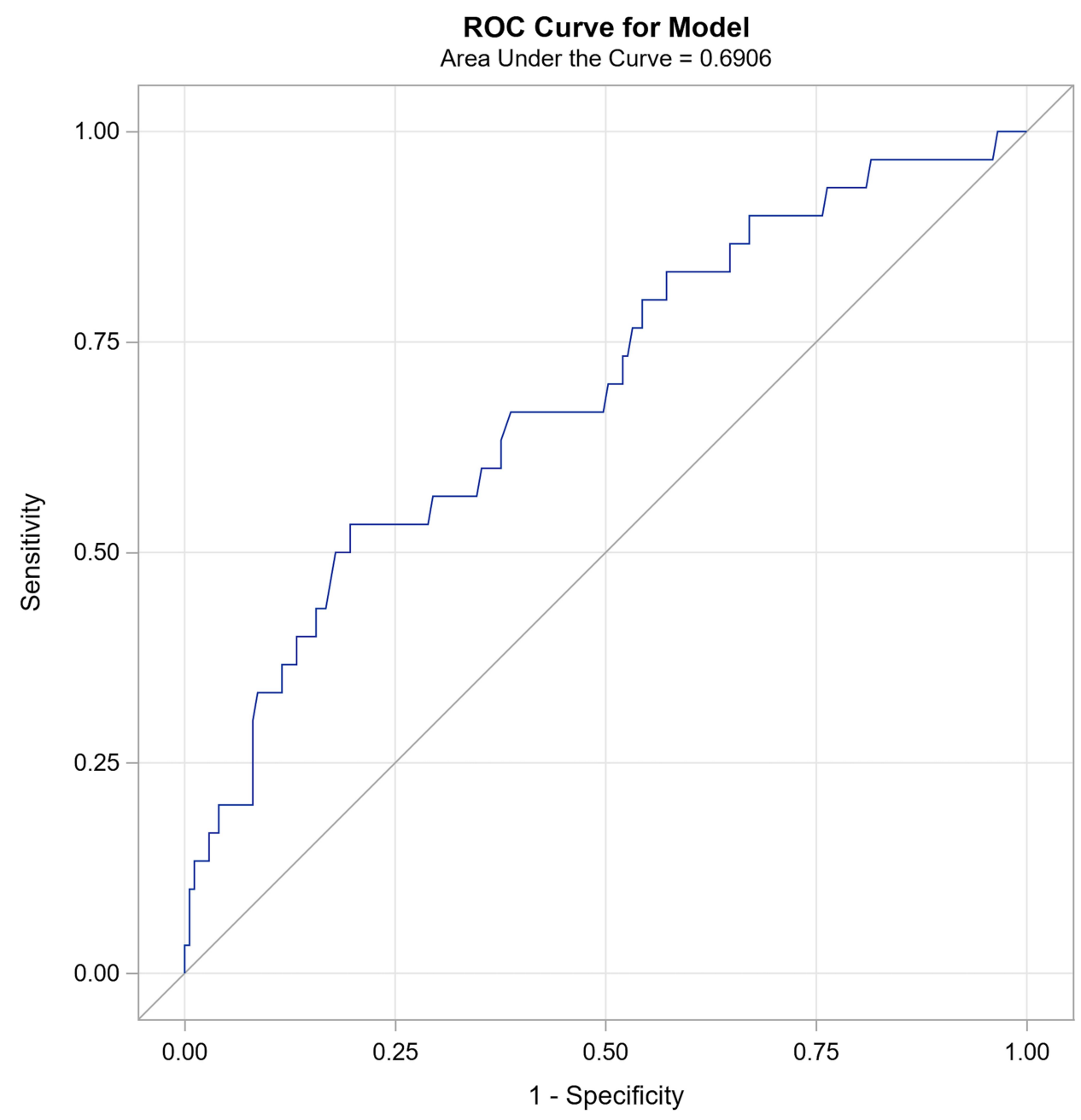

Figure 3 Receiver operating characteristic (ROC) of the suggested multivariate model for sub-acute post-cesarean pain.

Abbreviation: AUC, area under curve.

were little improvements in Akaike Information Criterion (AIC) compared to multivariable model without interaction term. Previous studies have engaged different QST to investigate post-cesarean neural sensitization such as electric pain threshold and pain tolerance thresholds, but threshold examinations are usually taken from a restricted area of pain, which may not be representative of the larger part of the somatosensory system. ${ }^{25}$ It was postulated that a long duration of cesarean delivery may lead to a more extensive incision and a lower dermatomal sensory level during anesthesia, and subsequently increased gain of the nociceptive system and enhanced neural sensitization. ${ }^{26}$ The use of CSI may hence be more clinically useful to quantify central sensitization due to its simplicity and less time-consuming nature as compared with other pain testing modalities.

The use of "three simple questions" to assess patients' anxiety about upcoming surgery, anticipated post-cesarean pain, and anticipated analgesic consumption was first reported by Pan et al, ${ }^{12}$ who found that answers to all these questions were modestly correlated with acute postcesarean pain scores $(\mathrm{r}=0.24-0.33, P<0.001)$. Here we showed that out of the three questions, only the anxiety about upcoming surgery was significantly associated with increased moderate-to-severe acute post-cesarean pain. A previous study has suggested differences in anticipated analgesia use in different races and ethnicities, ${ }^{27}$ and it is interesting that results reported in this study are different from the Pan et al study.

This study is one of the limited few prospective studies in an Asian population looking at multiple domains of demographic, pain, and psychological measurements. As compared with Chinese, Malay patients had an increased risk of having high acute 24 hours post-cesarean pain at rest. This is similar to the findings from a previous study 
by Tan et $\mathrm{al}^{28}$ in the same study site on women undergoing cesarean delivery, of which Malays were observed to have a higher mean pain score and higher morphine usage after surgery as compared with Chinese patients. Differences in culture and perception on this procedure, as well as genetic variant present or absent in different ethnicities may have contributed to the observed findings, however future research is warranted. We also collected information in a timely manner to determine how pre-operative factors could affect the post-cesarean pain not just in acute 24 hours post-cesarean delivery but also in a longer sub-acute term perspective.

However, we do acknowledge a number of limitations in our study. We enrolled only patients who were scheduled for elective cesarean delivery in our institution, and this may not be representative of the general obstetric population. Our study only selected patients who were to undergo elective cesarean delivery, many of whom had previous cesarean deliveries and hence may be more susceptible to neuropathic (entrapment of iliohypogastric or ilioinguinal nerves) and non-neuropathic (scar pain, pelvic pain, musculotendinous, dysmenorrhea, etc.) pain. ${ }^{29,30}$ There could also be confounders that were not taken into consideration which could contribute to higher pain profiles, such as lifestyle, social support, other pain, and psychometric measures (eg, fear avoidance, stress), which could be addressed in future studies. The pre-operative assessments were performed mainly by two investigators, with the question on LA injection before spinal anesthesia being performed with a standardized script to avoid variability. However, due to the pragmatic design of the study and the clinical settings, up to 15 attending anesthesiologists assigned to the operating theater were performing spinal anesthesia for different patients, which may introduce variations in terms of anesthetic techniques and decision-making, including non-standardized perioperative pain and anesthesia management. The recruited patients had their postnatal follow-up done at around 6 weeks after delivery, which might possibly delay the evaluation of sub-acute post-Cesarean pain. However, it is notable that the postnatal follow-up was done prospectively to investigate the presence of sub-acute post-cesarean pain that lasted for 4 weeks or more, which could occur any time after delivery till before the survey. In this study, we also assessed the postcesarean EPDS score, which is the gold standard in maternal mental health screening, with its outcome of postpartum depression having its onset starting at 4 weeks after delivery. It is also possible that some patients might have bias in memory recall, which may lead to varying follow-up outcomes from patient's timeliness to responding to the survey.

\section{Conclusion}

We showed that there was no association between increased pain score upon LA injection and acute post-cesarean pain, but it was associated with sub-acute post-cesarean pain. In addition, increased pain score upon LA injection and increased pre-operative central sensitization were associated with the presence of sub-acute post-cesarean pain at 6-10 weeks post-cesarean delivery. These findings may contribute to novel risk stratification strategies for post-cesarean pain management, however further research is warranted to validate these findings and to develop risk models.

\section{Abbreviations}

AIC, Akaike Information Criterion; ASA, American Society of Anesthesiologists; AUC, area under curve; BMI, body mass index; CI, confidence interval; CSE, combined spinalepidural; CSI, Central Sensitization Inventory; EPDS, Edinburgh Postnatal Depression Scale; HADS, Hospital Anxiety and Depression; IQR, interquartile range; LA, local anesthetic; NRS; numerical rating scale; OR, odds ratio; PCS, Pain Catastrophizing Scale; QST, quantitative sensory testing; ROC, receiver operating characteristic; $\mathrm{SD}$, standard deviation; TAP, transversus abdominis plane.

\section{Data Sharing Statement}

The datasets generated and analyzed in this work are available for anyone who wishes to access the data by contacting the corresponding author.

\section{Ethics Approval and Informed Consent}

The study was approved by the SingHealth Centralized Institutional Review Board, Singapore (SingHealth CIRB Ref: 2017/2381), and registered on Clinicaltrials.gov (NCT03645239). The authors declare that all the recruited patients provided informed consent, and that this work was conducted in accordance with the Declaration of Helsinki. All patients provided informed consent on the use of their de-identified data for publication purpose.

\section{Acknowledgments}

We would like to thank Ms Liu Juan (Senior Clinical Research Coordinator) and Ms Agnes Teo (Senior 
Clinical Research Coordinator) for their administrative support in this work.

\section{Funding}

This work was supported by the funding from the SingHealth Duke-NUS Academic Medical Centre, Anesthesiology and Perioperative Science Academic Clinical Program Pilot Research Grant (Grant no. ANAESPRG18/02). The aforementioned sponsor was not involved in the study activities.

\section{Disclosure}

All authors reported no conflicts of interest in this work.

\section{References}

1. Hamilton BE, Martin JA, Osterman MJ, Curtin SC, Mathews T. Births: final data for 2014. 2015.

2. James AH, Jamison MG, Brancazio LR, Myers ER. Venous thromboembolism during pregnancy and the postpartum period: incidence, risk factors, and mortality. Am J Obstet Gynecol. 2006;194 (5):1311-1315. doi:10.1016/j.ajog.2005.11.008

3. Eisenach JC, Pan PH, Smiley R, Lavand'homme P, Landau R, Houle TT. Severity of acute pain after childbirth, but not type of delivery, predicts persistent pain and postpartum depression. Pain. 2008;140(1):87-94. doi:10.1016/j.pain.2008.07.011

4. Niklasson B, Georgsson Öhman S, Segerdahl M, Blanck A. Risk factors for persistent pain and its influence on maternal wellbeing after cesarean section. Acta Obstet Gynecol Scand. 2015;94 (6):622-628. doi:10.1111/aogs.12613

5. Kainu JP, Sarvela J, Tiippana E, Halmesmäki E, Korttila K. Persistent pain after caesarean section and vaginal birth: a cohort study. Int J Obstet Anesth. 2010;19(1):4-9. doi:10.1016/j.ijoa.2009.03.013

6. Sng B, Sia A, Quek K, Woo D, Lim Y. Incidence and risk factors for chronic pain after caesarean section under spinal anaesthesia. Anaesth Intensive Care. 2009;37(5):748-752. doi:10.1177/0310057 X0903700513

7. Nikolajsen L, Sørensen H, Jensen T, Kehlet H. Chronic pain following caesarean section. Acta Anaesthesiol Scand. 2004;48(1):111-116. doi:10.1111/j.1399-6576.2004.00271.x

8. Ip HYV, Abrishami A, Peng PW, Wong J, Chung F. Predictors of postoperative pain and analgesic consumption: a qualitative systematic review. Anesthesiology. 2009;111(3):657-677.

9. Pan PH, Coghill R, Houle TT, et al. Multifactorial preoperative predictors for postcesarean section pain and analgesic requirement. Anesthesiology. 2006;104(3):417. doi:10.1097/00000542-20060300000007

10. Strulov L, Zimmer EZ, Granot M, Tamir A, Jakobi P, Lowenstein L. Pain catastrophizing, response to experimental heat stimuli, and postcesarean section pain. J Pain. 2007;8(3):273-279. doi:10.1016/j. jpain.2006.09.004

11. Orbach-Zinger S, Aviram A, Fireman S, et al. Severe pain during local infiltration for spinal anaesthesia predicts post caesarean pain. Eur J Pain. 2015;19(9):1382-1388. doi:10.1002/ejp.737
12. Pan PH, Tonidandel AM, Aschenbrenner CA, Houle TT, Harris LC, Eisenach JC. Predicting acute pain after cesarean delivery using three simple questions. Anesthesiology. 2013;118(5):1170-1179.

13. Landau R, Kraft JC, Flint LY, et al. An experimental paradigm for the prediction of Post-Operative Pain (PPOP). J Vis Exp. 2010;35:e1671.

14. Zaconeta AM, Queiroz I, Amato AA, Motta L, Casulari LA. Depression with postpartum onset: a prospective cohort study in women undergoing elective cesarean section in Brasilia, Brazil. Rev Bras Ginicol Obstet. 2013;35(3):130-135. doi:10.1590/S010072032013000300007

15. Van Tulder MW, Koes BW, Bouter LM. Conservative treatment of acute and chronic nonspecific low back pain: a systematic review of randomized controlled trials of the most common interventions. Spine. 1997;22(18):2128-2156. doi:10.1097/00007632-199709150-00012

16. Merskey HE. Classification of chronic pain: descriptions of chronic pain syndromés and definitions of pain terms. Pain. 1986.

17. Weissman-Fogel I, Granovsky Y, Crispel Y, et al. Enhanced presurgical pain temporal summation response predicts post-thoracotomy pain intensity during the acute postoperative phase. J Pain. 2009;10 (6):628-636. doi:10.1016/j.jpain.2008.12.009

18. Mayer TG, Neblett R, Cohen H, et al. The development and psychometric validation of the central sensitization inventory. Pain Pract. 2012;12(4):276-285. doi:10.1111/j.1533-2500.2011.00493.x

19. Cox J, Holden J. Perinatal Mental Health: A Guide to the Edinburgh Postnatal Depression Scale (EPDS). Royal College of Psychiatrists; 2003.

20. Zigmond AS, Snaith RP. The hospital anxiety and depression scale. Acta Psychiatr Scand. 1983;67(6):361-370. doi:10.1111/j.16000447.1983.tb09716.x

21. Sullivan MJ, Bishop SR, Pivik J. The pain catastrophizing scale: development and validation. Psychol Assess. 1995;7(4):524. doi:10.1037/1040-3590.7.4.524

22. Mathie A. Ion channels as novel therapeutic targets in the treatment of pain. J Pharm Pharmacol. 2010;62(9):1089-1095. doi:10.1111/ j.2042-7158.2010.01131.x

23. Hansson P, Backonja M, Bouhassira D. Usefulness and limitations of quantitative sensory testing: clinical and research application in neuropathic pain states. Pain. 2007;129(3):256-259. doi:10.1016/j.pain.2007.03.030

24. Eisenach J, Coghill R, Pan P, Harris L. Predictors of pain and analgesic use after cesarean section. Paper presented at: Anesthesiology; 2005.

25. Ang DC, Chakr R, France CR, et al. Association of nociceptive responsivity with clinical pain and the moderating effect of depression. J Pain. 2011;12(3):384-389. doi:10.1016/j.jpain.2010.09.004

26. Curatolo M, Arendt-Nielsen L, Petersen-Felix S. Central hypersensitivity in chronic pain: mechanisms and clinical implications. Phys Med Rehabil Clin. 2006;17(2):287-302. doi:10.1016/j.pmr.2005.12.010

27. Toledo P, Sun J, Grobman WA, Wong CA, Feinglass J, HasnainWynia R. Racial and ethnic disparities in neuraxial labor analgesia. Anesth Analg. 2012;114(1):172-178.

28. Tan E-C, Lim Y, Teo Y-Y, Goh R, Law H-Y, Sia AT. Ethnic differences in pain perception and patient-controlled analgesia usage for postoperative pain. J Pain. 2008;9(9):849-855. doi:10.1016/j. jpain.2008.04.004

29. Loos MJ, Scheltinga MR, Mulders LG, Roumen RM. The pfannenstiel incision as a source of chronic pain. Obstet Gynecol. 2008;111 (4):839-846.

30. Wang CB, Chiu WWC, Lee CY, Sun YL, Lin YH, Tseng CJ. Cesarean scar defect: correlation between cesarean section number, defect size, clinical symptoms and uterine position. Ultrasound Obstet Gynecol. 2009;34(1):85-89. doi:10.1002/uog.6405 
Journal of Pain Research

\section{Publish your work in this journal}

The Journal of Pain Research is an international, peer reviewed, open access, online journal that welcomes laboratory and clinical findings in the fields of pain research and the prevention and management of pain. Original research, reviews, symposium reports, hypothesis formation and commentaries are all considered for publication. The manuscript management system is completely online and includes a very quick and fair peer-review system, which is all easy to use. Visit http:// www.dovepress.com/testimonials.php to read real quotes from published authors. 